\title{
Tecnologias educativas empregadas na educação em saúde para pessoas com surdez:
}

\section{Uma revisão integrativa}

\author{
Educational technologies used in health education for people with deafness: An integrative review \\ Tecnologías educativas utilizadas en la educación sanitaria para personas con sordera: Una \\ revisión integradora
}

Recebido: 25/03/2021 | Revisado: 30/03/2021 | Aceito: 02/04/2021 | Publicado: 13/04/2021

Ana Karine Laranjeira de Sá
ORCID: https://orcid.org/0000-0003-4119-7076
Instituto Universitario Italiano de Rosario, Argentina
E-mail: aklenf@ hotmail.com
Marcella Tiburcio Maia
ORCID: https://orcid.org/0000-0003-3855-7771
E-mail: marcellamaia83@gmail.com
Alexiane Mendonça da Silva
Instituto Federal de Educação, Ciência e Tenologia de Penambuco, Brasil
ORCID: https://orcid.org/0000-0003-4000-1932
E-mail: alexiane.mendonca @ gmail.com
Andrés Cappelleti
Instituto Federal de Educação, Ciência e Tecnologia de Pernambuco, Brasil
ORCID: https://orcid.org/0000-0001-6854-4395
Instituto Universitario Italiano de Rosario, Argentina
E-mail: andrescappelletti1 @gmail.com

\section{Resumo}

A dificuldade de comunicação no indivíduo surdo traz sérias consequências dado aos obstáculos enfrentados para adquirir informação acerca de educação em saúde. Todavia, as tecnologias podem colaborar com o acesso ao conhecimento para a promoção de saúde e prevenção de agravos. Assim, o objetivo deste estudo é identificar evidências científicas encontradas na literatura acerca das tecnologias educacionais na área da saúde destinadas aos surdos. Tratase de uma revisão integrativa de literatura, realizada em seis etapas, em que os artigos foram selecionados em português, inglês e espanhol nas bases de dados: Medline via Pubmed, Literatura Latino-Americana e do Caribe em Ciências da Saúde, Cochrane Library e Web Of Science. Os artigos foram organizados por ano de publicação. A amostra final resultou em 10 artigos, publicados entre os anos de 2010 a 2020, com níveis de evidência II, III e IV. Na identificação dos recursos tecnológicos observados os vídeos educativos merecem destaque, utilizados em quatro (40\%) publicações, seguidos de avaliações no uso de sites em saúde, SMS, aplicativo e cursos on-line. Com isso, constatou-se a efetividade das tecnologias assistivas, as quais contribuem para a igualdade de acesso, qualidade de vida e aporte educacional para a promoção da saúde desse público.

Palavras-chave: Educação em saúde; Pessoas surdas; Tecnologias educativas.

\begin{abstract}
The difficulty of communication in the deaf individual has serious consequences given the obstacles faced to acquire information about health education. However, technologies can collaborate with access to knowledge for health promotion and disease prevention. Thus, the aim of this study is to identify scientific evidence found in the literature about educational technologies in the field of health for the deaf. It is an integrative literature review, carried out in six stages, in which the articles were selected in Portuguese, English and Spanish in the databases: Medline via Pubmed, Latin American and Caribbean Literature in Health Sciences, Cochrane Library and Web Of Science. The articles were organized by year of publication. The final sample resulted in 10 articles, published between 2010 and 2020, with levels of evidence II, III and IV. In the identification of the technological resources observed, educational videos deserve to be highlighted, used in four (40\%) publications, followed by evaluations in the use of health sites, SMS, application and online courses. With that, it was verified the effectiveness of assistive technologies, which contribute to equal access, quality of life and educational support for the promotion of the health of the public.
\end{abstract}

Keywords: Health education; Deaf people; Educational technologies. 


\begin{abstract}
Resumen
La dificultad de comunicación en el individuo sordo tiene graves consecuencias dados los obstáculos que enfrenta para adquirir información sobre educación para la salud. Sin embargo, las tecnologías pueden colaborar con el acceso al conocimiento para la promoción de la salud y la prevención de enfermedades. Así, el objetivo de este estudio es identificar las evidencias científicas encontradas en la literatura sobre tecnologías educativas en el campo de la salud para sordos. Se trata de una revisión bibliográfica integradora, realizada en seis etapas, en la que se seleccionaron los artículos en portugués, inglés y español en las bases de datos: Medline vía Pubmed, Literatura Latinoamericana y del Caribe en Ciencias de la Salud, Cochrane Library y Web Of Science. Los artículos se organizaron por año de publicación. La muestra final resultó en 10 artículos, publicados entre los años 2010 a 2020, con niveles de evidencia II, III y IV. En la identificación de los recursos tecnológicos observados, merecen destacarse los videos educativos, utilizados en cuatro (40\%) publicaciones, seguidos de evaluaciones en el uso de sitios de salud, SMS, aplicación y cursos en línea. Con eso, se verificó la efectividad de las tecnologías asistenciales, que contribuyen a la igualdad de acceso, calidad de vida y apoyo educativo para la promoción de la salud de este público.
\end{abstract}

Palabras clave: Educación para la salud; Personas sordas; Tecnologías educativas.

\title{
1. Introdução
}

A Organização Mundial da Saúde (OMS) define a deficiência como a perda ou a anormalidade de uma estrutura ou função psicológica, fisiológica ou anatômica. Entre as diversas deficiências, destaca-se a auditiva, pelo impacto que promove na vida social das pessoas. De acordo com a OMS, a perda auditiva é classificada de acordo com o limiar de audição verificado em decibéis (dBNA). Portanto, pode ser: leve (26 - $40 \mathrm{dBNA}$ ), moderada (41 - $60 \mathrm{dBNA})$, severa (61 - $80 \mathrm{dBNA}$ ) ou profunda (+ 80 dBNA) sendo classificada de três formas: bilateral, parcial ou total (OMS, 1997; França et al., 2016).

Considerada um problema mundial de saúde pública, a deficiência auditiva em escala global totaliza cerca de 360 milhões de pessoas surdas e segundo o Censo Demográfico de 2010 do Instituto Brasileiro de Geografia e Estatística (2021), o Brasil possui 9.717.318. Isso corresponde a um número significativo quando o enfoque é a saúde, pois mesmo a Língua Brasileira de Sinais (Libras) sendo considerada a segunda do país, muitos profissionais da saúde não possuem domínio sobre ela, dificultando muitas vezes os cuidados, apesar de sua utilização ser imprescindível para obtenção de uma comunicação efetiva e consequentemente, boa assistência (Gomes et al., 2017).

Atualmente, tem-se discutido muito sobre práticas para incluir socialmente essa população que, comumente, é vítima de preconceitos ou excluída da sociedade. Ao comparar os portadores de deficiência física, auditiva e visual, o surdo é quem se defronta com maior dificuldade de inclusão social, já que a audição é um sentido fundamental para obtenção e uso da linguagem e empregá-la. Percebe-se ainda, lacunas na preparação e conscientização dos profissionais da área da saúde e incentivo por parte dos governantes, com o intuito de capacitá-los para atenderem satisfatoriamente a essa população (Dantas et al., 2014).

Em meio a todos os avanços tecnológicos a tecnologia emprega-se como uma alternativa para auxiliar a comunicação da pessoa com surdez e facilitar a aprendizagem para promover sua saúde e prevenir agravos, além de gerar benefícios também para o profissional ao evitar equívocos no diagnóstico, dificuldades em elaborar o prontuário e tratamentos inadequados. Para isso, é imprescindível a inserção de tecnologias nos serviços de saúde, adesão dos profissionais e que essas tecnologias atendam as especificidades desse público (Galindo-Neto et al., 2019; Moreno et al., 2020; Soares et al., 2018).

Diante do exposto, mostra-se necessário no âmbito acadêmico, o estudo do uso das Tecnologias da Informação e Comunicação de modo democrático entre os surdos na área da saúde, para que este público possa alcançar uma melhor qualidade de vida, compreender de modo mais ampliado as problemáticas que concerne ao seu estado da saúde, prevenir de diversas formas as patologias e seguir com mais eficácia os tratamentos propostos.

Deste modo, o presente estudo tem como foco identificar evidências científicas encontradas na literatura acerca das tecnologias educacionais na área da saúde destinadas aos surdos, utilizando, como ponto de partida, a pergunta norteadora: "Quais tecnologias educativas são empregadas na educação em saúde para pessoas com surdez?" 


\section{Metodologia}

Trata-se de uma revisão integrativa da literatura nacional e internacional referente ao uso das tecnologias educativas em saúde destinadas aos surdos. Esse tipo de estudo compreende uma abordagem metodológica ampliada em relação a revisões de síntese, haja vista que permite que sejam combinados relatos da literatura teórica e empírica (Souza et al., 2010).

Esse estudo constituiu-se em seis etapas: 1) elaboração da pergunta da revisão; 2) busca e seleção dos estudos primários; 3) extração de dados dos estudos; 4) avaliação crítica dos estudos primários incluídos na revisão; 5) síntese dos resultados da revisão e 6) apresentação da revisão (Mendes et al., 2019).

Utilizou-se a estratégia PICo para delimitação da pergunta de pesquisa, em que P corresponde à População, I à Interesse e Co ao Contexto, desse modo, nesse estudo P são as pessoas surdas, I as tecnologias educativas e Co a educação em saúde, em sequência, estruturou-se a seguinte questão: Quais tecnologias educativas são empregadas na educação em saúde para pessoas com surdez?

Estabeleceu-se como critérios de inclusão: ser pesquisa acerca de tecnologia construída ou utilizada na educação em saúde de surdos, disponível nos idiomas inglês, espanhol ou português e publicados nos últimos 10 anos (período entre 2010 a 2020); e como critérios de exclusão: ser dissertação, tese, editorial, resumo em anais, relato de caso, artigo de revisão da literatura ou estudos que não sejam disponibilizados na íntegra. No caso dos artigos repetidos em mais de uma base, foram considerados para análise apenas um.

A busca dos artigos foi executada por dois avaliadores independentes em fevereiro de 2021, nas bases de dados: Medline via Pubmed, Literatura Latino-Americana e do Caribe em Ciências da Saúde (LILACS), Cochrane Library e Web Of Science, que foram selecionadas e acessadas por meio do Portal de Periódicos da Coordenação de Aperfeiçoamento de Pessoal de Nível Superior (CAPES) com o objetivo de obter-se uma quantidade maior de artigos.

Após a verificação dos Descritores Medical Subject Headings (Mesh), os seguintes cruzamentos foram efetuados com o uso do operador booleano AND: Persons With Hearing Impairments AND Technology; Persons With Hearing Impairments AND Health Education; Persons With Hearing Impairments AND Technology AND Health Education. As fases da busca foram seguidas e descritas baseadas no fluxograma da recomendação Principais Itens para Relatar Revisões sistemáticas e Metaanálises (PRISMA), como pode-se observar na Figura 1 (Galvão et al., 2015). 
Figura 1 - Fluxograma do processo de seleção dos artigos, baseado na recomendação PRISMA.

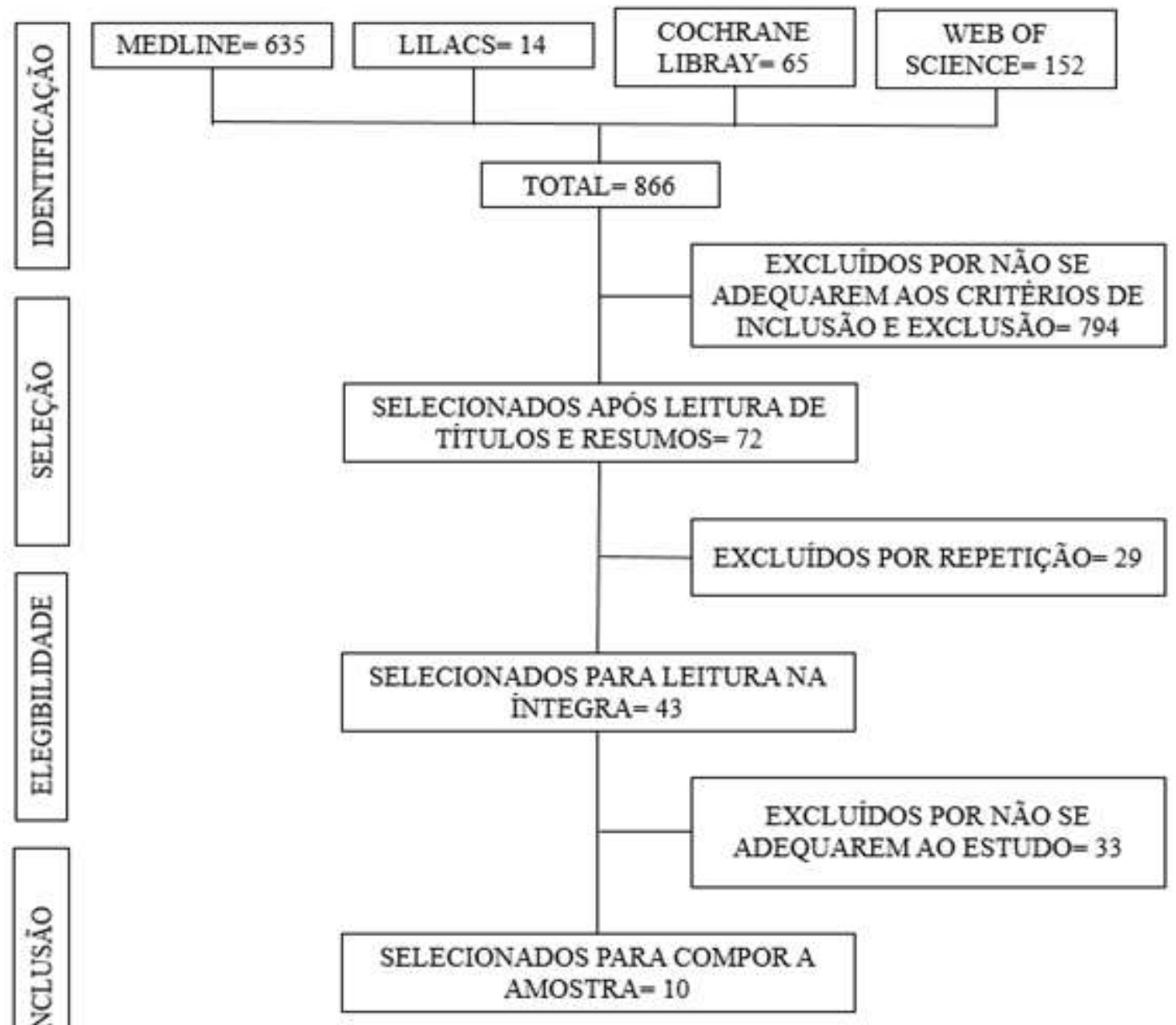

Fonte: Dados da pesquisa (2021).

Inicialmente na fase de identificação, foram constatados 866 artigos, 635 na Medline, 14 na LILACS, 65 na Cochrane Library e 152 na Web Of Science. Na fase de seleção foi realizado o refinamento dos achados, em que, deu-se a partir da leitura dos títulos e resumos de todos os trabalhos, excluindo os que, notoriamente, não se adequaram ao tema desta revisão ou aos critérios de inclusão e exclusão, assim, foram excluídos 794 estudos e 72 selecionados. Destes, excluíram-se 29 por repetição. Por conseguinte, na fase de elegibilidade foram selecionados 43 para leitura cuidadosa na íntegra dos estudos potencialmente elegíveis, excluindo-se 33 por apresentarem discordantes quanto à proposta deste estudo. E por fim, a fase de inclusão, que resultou em 10 estudos para compor a amostra.

Posteriormente para avaliar os estudos determinou-se o nível de evidência de acordo com esta classificação: nível I: metanálise de estudos controlados e randomizados; nível II: estudo experimental; nível III: estudo quase experimental; nível IV: estudo descritivo/não experimental ou com abordagem qualitativa; nível V: relato de caso ou experiência; nível VI: consenso e opinião de especialistas (Melnyk \& Fineout-Overholt, 2015).

No processo de extração e organização dos dados, os pesquisadores desenvolveram um instrumento próprio contemplando as variáveis: ano/ país/ idioma, autores/periódicos, público-alvo, tecnologia educativa, objetivos, principais resultados e conclusões e o nível de evidência. Os dados coletados foram dispostos em um quadro e organizados seguindo uma 
ordem cronológica, do mais recente ao mais antigo, em que se considerou o ano de publicação, nos quais, os resultados foram sintetizados, apresentados e discutidos na seção seguinte.

Em síntese, por tratar-se de revisão integrativa, a pesquisa não foi submetida ao Comitê de Ética em Pesquisa, porém foram mantidas as ideias dos autores das publicações utilizadas no desenvolvimento deste estudo.

\section{Resultados e Discussão}

Foram selecionados 10 artigos que abordavam tecnologias educativas voltadas à promoção da educação em saúde para a população surda. Evidenciou-se um aumento do número de publicações nos anos de 2017 a 2019, em que dois (20\%) estudos foram publicados a cada ano, sendo o inglês a língua preponderante, registrados em oito (80\%) das publicações, seguidas do português com dois (20\%). O tema mais abordado foi o câncer, com cinco (50\%) artigos, seguidos de autogerenciamento da perda auditiva, reanimação cardiorrespiratória, educação em saúde sexual e reprodutiva, hipertensão arterial e acessibilidade de sites com informação em saúde, com uma (10\%) publicação cada tema.

O periódico de publicação mais frequente foi o Journal of Cancer Education com quatro (40\%) artigos. A descrição da população mais estudada, foi pessoas surdas, com ocorrência em cinco (50\%) artigos, seguidos de surdos com mais de 18 anos, surdos com mais de 50 anos, estudantes surdos, homens surdos, homens e mulheres que se identificavam como surdos, um (10\%) para cada. Os Estados Unidos foi o país que realizou mais estudos, sete (70\%) deles, com sequência tem-se Brasil com dois (20\%) e África do Sul com um (10\%).

Quanto aos tipos de estudos registrados, contabilizou: cinco (50\%) quase-experimentais (nível de evidência III); três (30\%) experimentais (nível de evidência II); e dois (20\%) foram descritivos (nível de evidência IV). Na identificação dos recursos tecnológicos observados os vídeos educativos merecem destaque, utilizados em quatro (40\%) estudos, seguidos de três (30\%) avaliações no uso de sites em saúde, SMS, aplicativo e cursos on-line, com um (10\%) para cada.

No Quadro 1 observa-se a caracterização dos estudos selecionados nesta revisão, em que se apresentou os resultados acima citados e os demais dados coletados.

Quadro 1 - Caracterização dos estudos selecionados nesta revisão. Arcoverde, PE, Brasil, 2021.

\begin{tabular}{|c|c|c|c|c|c|c|}
\hline $\begin{array}{l}\text { Ano/ País/ } \\
\text { Idioma }\end{array}$ & $\begin{array}{l}\text { Autores/ } \\
\text { Periódico }\end{array}$ & $\begin{array}{l}\text { Público- } \\
\text { alvo }\end{array}$ & $\begin{array}{l}\text { Tecnologia } \\
\text { educativa }\end{array}$ & Objetivos & $\begin{array}{lll}\text { Principais } & \text { resultados } & \text { e } \\
\text { conclusões } & & \end{array}$ & $\begin{array}{l}\text { Nível de } \\
\text { evidência }\end{array}$ \\
\hline $\begin{array}{l}\text { 2020/ } \\
\text { Estados } \\
\text { Unidos/ } \\
\text { Inglês }\end{array}$ & $\begin{array}{l}\text { Pronk et al/ } \\
\text { American } \\
\text { Journal of } \\
\text { Audiology }\end{array}$ & \begin{tabular}{ll}
\multicolumn{2}{l}{ Adultos } \\
surdos \\
com & 50 \\
anos & ou \\
mais &
\end{tabular} & Aplicativo & $\begin{array}{lr}\text { Desenvolver } & \text { e } \\
\text { avaliar } & \text { um } \\
\text { aplicativo } & \text { de } \\
\text { smartphone } & \\
\text { personalizado } \\
\text { projetado para } \\
\text { melhorar } \\
\text { autogerenciament } \\
\text { o da perda auditiva } \\
\text { e fornecer uma } \\
\text { alternativa inicial } \\
\text { para a reabilitação } \\
\text { de HÁ (aparelho } \\
\text { auditivo). }\end{array}$ & $\begin{array}{l}\text { Intervenções adaptativas in- } \\
\text { time que são oferecidas via } \\
\text { celular aplicativos de saúde, } \\
\text { como o aplicativo HEAR- } \\
\text { aware, pode encorajar as } \\
\text { pessoas a autogerirem sua } \\
\text { saúde auditiva fora das portas } \\
\text { de uma clínica de audição ou } \\
\text { loja de aparelhos auditivos e } \\
\text { tem potencial para manter ou } \\
\text { aumentar o seu nível de } \\
\text { participação social. }\end{array}$ & II \\
\hline
\end{tabular}




\begin{tabular}{|c|c|c|c|c|c|c|}
\hline $\begin{array}{l}\text { 2019/ } \\
\text { Brasil/ } \\
\text { Português }\end{array}$ & $\begin{array}{l}\text { Galindo-Neto et } \\
\text { al/ Revista } \\
\text { Latino } \\
\text { Americana de } \\
\text { Enfermagem }\end{array}$ & $\begin{array}{l}\text { Alunos } \\
\text { surdos }\end{array}$ & Vídeo & $\begin{array}{l}\text { Construir e validar } \\
\text { vídeo educativo } \\
\text { para ensino de } \\
\text { alunos surdos } \\
\text { acerca } \\
\text { ressuscitação da } \\
\text { cardiopulmonar. }\end{array}$ & $\begin{array}{l}\text { O vídeo foi considerado } \\
\text { como tendo conteúdo válido } \\
\text { pelos juízes e conteúdo } \\
\text { compreensível pelos alunos } \\
\text { surdos. Dessa forma, o vídeo } \\
\text { representa uma tecnologia } \\
\text { inclusiva para educação em } \\
\text { saúde de surdos sobre } \\
\text { ressuscitação } \\
\text { cardiopulmonar. }\end{array}$ & IV \\
\hline $\begin{array}{l}2019 / \\
\text { Estados } \\
\text { Unidos/ } \\
\text { Inglês }\end{array}$ & $\begin{array}{l}\text { Engelberg et al/ } \\
\text { Journal of } \\
\text { Cancer } \\
\text { Education }\end{array}$ & Surdos & Sites & $\begin{array}{l}\text { Avaliar o grau em } \\
\text { que o } \\
\text { emparelhamento } \\
\text { de mensagens de } \\
\text { saúde com humor } \\
\text { pode facilitar o } \\
\text { aumento do } \\
\text { conhecimento } \\
\text { sobre câncer, a } \\
\text { retenção desse } \\
\text { conhecimento } \\
\text { adquirido; } \\
\text { promoção pessoal } \\
\text { do site } \\
\text { educacional } \\
\text { amigável para } \\
\text { surdos, e as visitas } \\
\text { repetidas ao site. }\end{array}$ & $\begin{array}{l}\text { Foram demonstrados em } \\
\text { quase todas as medidas, } \\
\text { incluindo um aumento } \\
\text { sustentado no } \\
\text { comportamento de busca de } \\
\text { informações sobre o câncer e } \\
\text { a intenção de melhorar os } \\
\text { hábitos de saúde. A maioria } \\
\text { dos participantes relatou que } \\
\text { ficaria motivada a retornar a } \\
\text { esse site e encaminhá-lo a } \\
\text { outras pessoas, desde que } \\
\text { fosse atualizado } \\
\text { regularmente com novas } \\
\text { piadas. }\end{array}$ & III \\
\hline $\begin{array}{l}2018 / \\
\text { Estados } \\
\text { Unidos/ } \\
\text { Inglês }\end{array}$ & $\begin{array}{l}\text { Kushalnagar et } \\
\text { al/ Journal of } \\
\text { Cancer } \\
\text { Education }\end{array}$ & $\begin{array}{l}\text { Pessoas } \\
\text { surdas }\end{array}$ & Sites & $\begin{array}{lr}\text { Explorar } & \text { o } \\
\text { benefício } & \text { da } \\
\text { simplificação do } \\
\text { texto em língua } \\
\text { americana de } \\
\text { sinais sobre a } \\
\text { saúde do câncer na } \\
\text { Internet. }\end{array}$ & $\begin{array}{l}\text { Os resultados indicaram que } \\
\text { estudantes universitários } \\
\text { ouvintes se saíram bem tanto } \\
\text { na versão original quanto na } \\
\text { simplificada. A compreensão } \\
\text { de estudantes universitários } \\
\text { surdos, em contraste, } \\
\text { beneficiou } \\
\text { significativamente do texto } \\
\text { simplificado. }\end{array}$ & III \\
\hline $\begin{array}{l}\text { 2017/ } \\
\text { África do } \\
\text { Sul/ Inglês }\end{array}$ & $\begin{array}{l}\text { Haricharan et al/ } \\
\text { BMC Public } \\
\text { Health }\end{array}$ & $\begin{array}{l}\text { Surdos } \\
\text { com mais } \\
\text { de } 18 \text { anos }\end{array}$ & $\begin{array}{l}\text { SMS } \\
\text { (Serviço de } \\
\text { mensagem } \\
\text { curta) }\end{array}$ & $\begin{array}{l}\text { Avaliar a melhoria } \\
\text { de conhecimento } \\
\text { dos surdos sobre } \\
\text { hipertensão } \\
\text { arterial e vida } \\
\text { saudável através } \\
\text { de uma campanha } \\
\text { de promoção à } \\
\text { saúde baseada em } \\
\text { SMS. }\end{array}$ & $\begin{array}{l}\text { SMSs foram eficazes para } \\
\text { melhorar o conhecimento das } \\
\text { pessoas surdas sobre } \\
\text { hipertensão e vida saudável. } \\
\text { No entanto, as campanhas de } \\
\text { SMS devem estar cientes das } \\
\text { necessidades exclusivas e } \\
\text { preferências de comunicação } \\
\text { das pessoas surdas e explorar } \\
\text { como acomodá-las. }\end{array}$ & III \\
\hline
\end{tabular}




\begin{tabular}{|c|c|c|c|c|c|c|}
\hline $\begin{array}{l}2017 / \\
\text { Estados } \\
\text { Unidos/ } \\
\text { Inglês }\end{array}$ & $\begin{array}{l}\text { Palmer et al/ } \\
\text { Disabil Health }\end{array}$ & Surdos & Vídeo & $\begin{array}{lr}\begin{array}{l}\text { Comparar } \\
\text { eficácia }\end{array} & \text { a } \\
\text { informações } & \\
\text { genéticas } & \text { do } \\
\text { câncer } & \\
\text { apresentadas } & \\
\text { usando } & \\
\text { abordagem } & \\
\text { bilíngue (Língua } \\
\text { americana } \\
\text { sinais ASL } & \text { com } \\
\text { legendas } \\
\text { inglês) em } \\
\text { abordagem } \\
\text { monolíngue (texto } \\
\text { em inglês). }\end{array}$ & $\begin{array}{l}\text { Uma abordagem online } \\
\text { permite que pessoas surdas } \\
\text { em qualquer área geográfica } \\
\text { acessem informações } \\
\text { genéticas importantes. Um } \\
\text { formato bilíngue usando } \\
\text { videoclipes ASL com opções } \\
\text { de legenda oculta fornece a } \\
\text { essa população acesso total } \\
\text { em seu idioma preferido com } \\
\text { acesso a terminologia médica } \\
\text { específica em inglês escrito. }\end{array}$ & II \\
\hline $\begin{array}{l}\text { 2016/ } \\
\text { Brasil/ } \\
\text { Português }\end{array}$ & $\begin{array}{l}\text { Áfio et al/ } \\
\text { Revista } \\
\text { Brasileira de } \\
\text { Enfermagem }\end{array}$ & Surdos & $\begin{array}{l}\text { Curso on- } \\
\text { line }\end{array}$ & $\begin{array}{l}\text { Avaliar } \\
\text { acessibilidade } \\
\text { automática de } \\
\text { tecnologia } \\
\text { assistiva, na } \\
\text { modalidade de } \\
\text { curso on-line, para } \\
\text { surdos acerca da } \\
\text { Educação em } \\
\text { Saúde Sexual e } \\
\text { Reprodutiva: o uso } \\
\text { de preservativos. }\end{array}$ & $\begin{array}{l}\text { Conclui-se que as páginas do } \\
\text { curso foram consideradas, } \\
\text { pelo software utilizado, } \\
\text { adequadas aos padrões de } \\
\text { acessibilidade na Web. }\end{array}$ & I) \\
\hline $\begin{array}{l}2015 / \\
\text { Estados } \\
\text { Unido/ } \\
\text { Inglês }\end{array}$ & $\begin{array}{l}\text { Kushalnagar et } \\
\text { al/ Health } \\
\text { Communication }\end{array}$ & Surdos & $\begin{array}{l}\text { Sites com } \\
\text { vídeos }\end{array}$ & $\begin{array}{l}\text { Verificar } \\
\text { usabilidade de } \\
\text { quatro sites de } \\
\text { saúde que incluem } \\
\text { vídeos em língua } \\
\text { americana de } \\
\text { sinais (ASL). }\end{array}$ & $\begin{array}{l}\text { Os participantes com menor } \\
\text { nível de alfabetização em } \\
\text { saúde tiveram maior } \\
\text { dificuldade em encontrar } \\
\text { informações em sites de } \\
\text { saúde acessíveis à ASL. A } \\
\text { preferência dos participantes } \\
\text { é por um site de saúde ideal } \\
\text { acessível à ASL e conclui } \\
\text { com uma discussão sobre o } \\
\text { papel dos sites acessíveis na } \\
\text { promoção da alfabetização } \\
\text { em saúde em usuários de } \\
\text { ASL. }\end{array}$ & II \\
\hline
\end{tabular}




\begin{tabular}{|c|c|c|c|c|c|c|}
\hline $\begin{array}{l}2013 / \\
\text { Estados } \\
\text { Unidos/ } \\
\text { Inglês }\end{array}$ & $\begin{array}{lll}\text { Sacks et al/ } & \text { Journal } \\
\text { Cancer } & & \\
\text { Education } & \end{array}$ & $\begin{array}{l}\text { Homens } \\
\text { surdos }\end{array}$ & Vídeo & $\begin{array}{l}\text { Avaliar o valor } \\
\text { educacional do } \\
\text { vídeo sobre câncer } \\
\text { testicular. }\end{array}$ & $\begin{array}{l}\text { Embora os homens que } \\
\text { ouvem tenham pontuações } \\
\text { mais altas no pré-teste do que } \\
\text { os surdos, tanto os surdos } \\
\text { quanto os que ouvem } \\
\text { demonstraram aumentos } \\
\text { significativos nos escores de } \\
\text { conhecimento geral do } \\
\text { câncer testicular após assistir } \\
\text { ao vídeo. }\end{array}$ & II \\
\hline $\begin{array}{l}2012 / \\
\text { Estados } \\
\text { Unidos/ } \\
\text { Inglês }\end{array}$ & $\begin{array}{ll}\begin{array}{l}\text { Harry et } \\
\text { Journal } \\
\text { cancer }\end{array} & \text { of } \\
\text { education } & \end{array}$ & $\begin{array}{l}\text { Homens e } \\
\text { mulheres } \\
\text { que se } \\
\text { identificar } \\
\text { am como } \\
\text { membros } \\
\text { da comunida } \\
\text { de de } \\
\text { surdos }\end{array}$ & Vídeo & $\begin{array}{l}\text { Avaliar um vídeo } \\
\text { educacional sobre } \\
\text { câncer de pele em } \\
\text { ASL desenvolvido } \\
\text { especificamente } \\
\text { para a comunidade } \\
\text { surda. }\end{array}$ & $\begin{array}{l}\text { Demonstrou que fornecer } \\
\text { informações sobre o controle } \\
\text { do câncer de pele por meio de } \\
\text { um vídeo voltado para surdos } \\
\text { com alinhamento linguístico } \\
\text { e cultural pode aumentar o } \\
\text { conhecimento dos } \\
\text { espectadores sobre as opções } \\
\text { de controle do câncer de pele, } \\
\text { reforçando as conclusões dos } \\
\text { estudos anteriores da equipe } \\
\text { de pesquisa. }\end{array}$ & II \\
\hline
\end{tabular}

Fonte: Dados da pesquisa (2021).

Verifica-se que, dentre as tecnologias que mais favorecem a educação em saúde e acessibilidade dos surdos estão os vídeos didáticos. Galindo-Neto et al. (2019) trazem um vídeo sobre ressuscitação pulmonar, onde foi avaliado mediante o critério de validação com a concordância que foi superior a 80\%, analisado por meio do índice de validação de conteúdo e teste binomial entre alunos e professores surdos, evidenciou-se que essa ferramenta foi útil na questão pedagógica e teve boa aceitabilidade entre os surdos.

Kushalnagar et al. (2018) criaram um mecanismo de simplificação da linguagem na língua de sinais permitindo a simplificação do texto sem alteração do conteúdo, na área de câncer. A pesquisa mostrou uma boa aceitabilidade e entendimento no caso de indivíduos surdos leigos. Tal fato demonstra a importância de usar, além de uma linguagem adequada, uma forma mais simplificada, mantendo-se a essência do texto, a fim de alcançar tal público.

Kushalnagar et al. (2015) trazem ao debate a relevância da alfabetização em língua de sinais, haja vista que nos experimentos realizados foi constatado que surdos com dificuldades na língua de sinais conseguem apenas uma baixa interação com os sites na área de saúde. Portanto, a aprendizagem da língua de sinais é essencial para que os surdos possam avançar nos conhecimentos na área de saúde.

Sacks et al. (2013) constataram que embora a língua de sinais seja o método predominante de comunicação dentro da comunidade surda, muitos membros da comunidade possuíam alfabetização suficiente em inglês para usar legendas abertas e complementar a assimilação de informações assinadas complexas, tornando-se culturalmente apropriado oferecer versões tanto assinadas quanto legendadas versões do script. Portanto, legendas abertas também foram incluídas no vídeo, juntamente com uma narração do script em libras, ambos os quais possibilitaram que pessoas surdas assistissem ao vídeo com familiares e amigos ouvintes e com deficiência auditiva. Essa ferramenta possibilita a interação dos surdos com não surdos, possibilitando a equidade entre os grupos.

Palmer et al. (2017) também realizaram um estudo semelhante referente à exposição de vídeo sobre câncer testicular e 
de próstata. O vídeo foi transmitido na língua americana de sinais (ASL) com legenda em inglês e de forma monolíngue somente em inglês, onde foi contatado que a modalidade bilíngue dá mais credibilidade ao público de surdos, ainda que estes possuam uma escolaridade básica.

Enfatiza-se a questão da interatividade, haja vista que o surdo, além de obter conhecimentos sobre saúde, tem a condição de assistir ao vídeo com a família, e assim, interagir e trocar ideias acerca do conteúdo aprendido, gerando uma socialização que beneficia o núcleo familiar, e não apenas o surdo. Fato esse constatado também por Sacks et al. (2013).

Harry et al. (2012) executaram um experimento comparando a eficácia das informações genéticas on-line do câncer apresentadas usando uma abordagem bilíngue (ASL com legendas em inglês) e uma abordagem monolíngue (texto em inglês) sobre câncer de pele, onde foi constatado que os participantes que assistiram o vídeo sobre câncer de pele ganharam muito mais conhecimento do que os participantes de controle, demonstrando a eficácia do vídeo em aumentar o conhecimento sobre a temática.

Portanto, a utilização de vídeos educativos em saúde desperta interesse, ampliam o acesso às informações em saúde, aproximam o público das necessidades educativas específicas, promovem autonomia, inclusão social e consequentemente melhoram a qualidade de vida da população surda.

Além dos vídeos, também foram averiguados os recursos do aplicativo de smarthphone para melhorar o autogerenciamento da perda auditiva, um score referente a mensagens de saúde utilizando o humor, um software que detecta falhas no uso com os surdos, e ainda o uso de SMS sobre hipertensão para surdos.

Dentre as tecnologias estudadas, Pronk et al. (2020) colocou a relevância da autogestão da deficiência auditiva pela utilização de um aplicativo de smartphone, onde o conteúdo é adaptado ao estágio dinâmico de prontidão de uma pessoa para tomar medidas em sua audiência (estágio de mudança) e para situações acústicas dinâmicas da pessoa e desafios associados, sendo este um aparelho de intervenção, sendo a gestão de responsabilidade do usuário com deficiência.

Áfio et al. (2016), traz também uma tecnologia onde os surdos podem utilizar a autogestão, que é a proposta de avaliação de acessibilidade automática de tecnologia assistiva em cursos online para surdos. O curso colocado para os surdos teve como temática o uso dos preservativos, onde é avaliada automaticamente a questão da acessibilidade. Essa ferramenta é muito útil pois detecta as falhas para que sejam realizados possíveis ajustes no software, para que a linguagem esteja cada vez mais acessível e compreensível ao grupo de surdos.

Harichagan et al. (2017) executaram uma experiência com o uso de SMS sobre informações acerca de hipertensão para surdos devido ao uso comum de aparelhos celulares no local de estudo, na África do Sul. A campanha teve como objetivo colocar a relevância do cuidado e atenção com a saúde dos hipertensos, e mostrou que essa ferramenta pode ser facilmente manejada pelos surdos. Portanto, é preciso que sejam investigadas nesse âmbito do uso das tecnologias educacionais, a aceitação dos indivíduos surdos por determinadas tecnologias, como por exemplo, o aparelho celular, como nesse caso.

Engelberg et al. (2019) trouxeram a contribuição de um site sobre câncer na língua americana de sinais com informações pautadas no humor, o que agradou a maior parte dos leitores, que afirmaram que voltariam a acessar o site, caso o mesmo continuasse a ser atualizado desse modo. Essa inovação mostra que o público surdo está aberto tanto a novas tecnologias quanto ao não tradicional na área de saúde. Além disso, ajustes são realizados à medida que detectados com o objetivo de aprimorar a tecnologia para o uso dos surdos.

Evidencia-se o uso da tecnologia por meio de softwares e aplicativos destinados ao grupo de surdos tanto para uso (Pronk et al., 2020; Áfio et al. 2016; Haricharan et al., 2017) como para monitoramento de ajustes (Engelberg et al., 2019), permitindo um avanço na interação e melhoramento da tecnologia de acordo com as demandas dos surdos. Entretanto, o grande desafio com as tecnologias assistivas é torná-las acessíveis aos surdos, de modo a promover a inclusão e igualdade entre o público surdo através das TICs, utilizá-los como meio para comunicação e adequar a linguagem para a língua de sinais. 
Portanto, o uso das supracitadas tecnologias assistivas permite a comunicação e interação entre surdos e deficientes auditivos, sendo eficazes na educação em saúde, ao possibilitar ampliar o conhecimento em saúde dos surdos e propiciar ajustes cada vez mais eficazes e tecnológicos capazes de estimular mudanças de comportamento para a melhoria dos hábitos de saúde, motivar a busca de conhecimentos para suprir as necessidades específicas da limitação da linguagem.

\section{Considerações Finais}

O estudo demonstrou os resultados positivos da utilização das TICs na educação em saúde para pessoas surdas, houve predominância das estratégias com vídeo, mas também êxito com aplicativos, sites, cursos on-line e SMS. O conteúdo mais abordado foi o câncer, a maioria realizou o tipo de estudo quase experimental e constata a efetividade das tecnologias assistivas para o propósito aqui averiguado, a qual contribui para a igualdade de acesso, qualidade de vida e aporte educacional para a promoção da saúde desse público.

Como lacunas de conhecimento, verifica-se poucos estudos sobre o desenvolvimento de tecnologias assistivas em saúde, quantidade pequena de temas versados para este público, ausência de estudos com associação de tecnologias educacionais.

Portanto, como sugestão para posteriores estudos o desenvolvimento de tecnologias educativas para o público surdo em diversas temáticas, associar abordagens tecnológicas diferentes e provar sua efetividade a pequeno e a longo prazo.

\section{Referências}

Áfio, A. C. E., Carvalho, A. T. D., Carvalho, L. V. D., Silva, A. S. R. D., \& Pagliuca, L. M. F. (2016). Accessibility assessment of assistive technology for the hearing impaired. Revista brasileira de enfermagem, 69(5), 833-839. http://dx.doi.org/10.1590/0034-7167.2016690503

Dantas, T. R. A, Gomes, T. M., Costa, T. F., Azevedo, T. R., Brito, S. S., \& Costa, K. N. F. M. (2014). Comunicação entre a equipe de enfermagem e pessoas com deficiência auditiva. Revista Enfermagem UERJ, 22(2), 169-174. https://www.e-publicacoes.uerj.br/index.php/enfermagemuerj/article/view/13559/10365

Engelberg, M., Nakaji, M. C., Harry, K. M., Wang, R. M., Kennedy, A., Pan, T. M., Sanchez, T., \& Sadler, G. R. (2019). Promotion of Healthy Humor Cancer Education Messages for the Deaf Community. Journal of cancer education: the official journal of the American Association for Cancer Education, 34(2), 323328. https://doi-org.ez360.periodicos.capes.gov.br/10.1007/s13187-017-1305-5

França, E. G., Pontes, M. A., Costa, G. M. C., \& França, I. S. X. (2016). Dificuldades de profissionais na atenção à saúde da pessoa com surdez severa. Ciencia y enfermería, 22(3), 107-116. https://dx.doi.org/10.4067/S0717-95532016000300107

Galindo Neto, N. M., Áfio, A. C. E., Leite, S. D. S., Silva, M. G. D., Pagliuca, L. M. F., \& Caetano, J. Á. (2019). Tecnologias para educação em saúde de surdos: revisão integrativa. Texto \& Contexto-Enfermagem, 28. https://www.scielo.br/pdf/tce/v28/pt_1980-265X-tce-28-e20180221.pdf

Galindo-Neto, N. M., Alexandre, A. C. S., Barros, L. M., Sá, G. G. M., Carvalho, K. M., \& Caetano, J. Á. (2019). Criação e validação de um vídeo educativo para surdos sobre ressuscitação cardiopulmonar. Revista Latino-Americana de Enfermagem, 27, e3130. https://doi.org/10.1590/1518-8345.2765.3130

Galvão, T. F., Pansani, T. D. S. A., \& Harrad, D. (2015). Principais itens para relatar Revisões sistemáticas e Meta-análises: A recomendação PRISMA. Epidemiologia e Serviços de Saúde, 24, 335-342. https://www.scielo.br/pdf/ress/v24n2/2237-9622-ress-24-02-00335.pdf

Gomes, L. F., Machado, F. C., Lopes, M. M., Oliveira, R. S., Medeiros-Holanda, B., Silva, L. B., Barletta, J. B. \& Kandratavicius, L. (2017). Conhecimento de Libras pelos médicos do Distrito Federal e atendimento ao paciente surdo. Revista brasileira de educação médica, 41(4), 551-556. https://dx.doi.org/10.1590/1981-52712015v41n3rb20160076

Haricharan, H. J., Heap, M., Hacking, D., \& Lau, Y. K. (2017). A promoção da saúde via SMS melhora o conhecimento sobre hiper tensão para surdos sulafricanos. BMC public health, 17 (1), 663. https://doi.org/10.1186/s12889-017-4619-7

Harry, K. M., Malcarne, V. L., Branz, P., Fager, M., Garcia, B. D., \& Sadler, G. R. (2012). Evaluating a skin cancer education program for the deaf community. Journal of Cancer Education, 27(3), 501-506. https://www.ncbi.nlm.nih.gov/pmc/articles/PMC4153386/

Instituto Brasileiro de Geografia Estatística (2021). Censo demográfico 2010: Estatísticas de gênero: Deficiência auditiva. https://www.ibge.gov.br/apps/snig/v1/?loc=0\&cat=-1,-2,-3,128\&ind=4643

Kushalnagar, P., Naturale, J., Paludneviciene, R., Smith, S. R., Werfel, E., Doolittle, R., Jacobs, S. \& DeCaro, J. (2015). Health websites: Accessibility and usability for American Sign Language users. Health communication, 30(8), 830-837. https://doi.org/10.1080/10410236.2013.853226

Kushalnagar, P., Smith, S., Hopper, M., Ryan, C., Rinkevich, M., \& Kushalnagar, R. (2018). Making cancer health text on the Internet easier to read for deaf people who use American Sign Language. Journal of Cancer Education, 33(1), 134-140. https://pubmed.ncbi.nlm.nih.gov/27271268/

Melnyk, B. M., \& Fineout-Overholt, E. (Eds.). (2015). Evidence-based practice in nursing \& healthcare: A guide to best practice (3rd ed.). Lippincott Williams \& Wilkins. 
Research, Society and Development, v. 10, n. 4, e345410414287, 2021

(CC BY 4.0) | ISSN 2525-3409 | DOI: http://dx.doi.org/10.33448/rsd-v10i4.14287

Mendes, K. D. S., Silveira, R. C. D. C. P., \& Galvão, C. M. (2019). Uso de gerenciador de referências bibliográficas en la selección de los estudios primarios en revisión integrativa. Texto \& Contexto-Enfermagem, 28. http://www.scielo.br/scielo.php?script=sci_arttext\&pid=S0104$07072019000100602 \& \operatorname{lng}=\mathrm{en} \& \mathrm{nrm}=$ iso

Palmer, C. G., Boudreault, P., Berman, B. A., Wolfson, A., Duarte, L., Venne, V. L., \& Sinsheimer, J. S. (2017). Bilingual approach to online cancer genetics education for Deaf American Sign Language users produces greater knowledge and confidence than English text only: A randomized study. Disability and health journal, 10(1), 23-32. https://pubmed.ncbi.nlm.nih.gov/27594054/

Pollard Jr, R. Q., Dean, R. K., O'Hearn, A., \& Haynes, S. L. (2009). Adapting health education material for deaf audiences. Rehabilitation psychology, 54(2), 232-238. https://doi.org/10.1037/a0015772

Pronk, M., Besser, J., Smits, C., Feenstra-Kikken, V., van Beek, H., Polleunis, C., \& Kramer, S. E. (2020). Rationale, theoretical underpinnings, and design of HEAR-aware: Providing adults with hearing loss with tailored support to self-manage their hearing problems via a smartphone app, as an alternative to hearing aids. American Journal of Audiology, 29(3S), 648-660. https://doi.org/10.1044/2020_AJA-19-00079

Moreno, R. S. R., Silva, N. C. S., Oliveira, V. S., \& da Silva, J. G. (2020). Tecnologias assistivas na comunicação de pacientes com deficiência auditiva em serviços de saúde no Brasil. Brazilian Journal of https://www.brazilianjournals.com/index.php/BRJD/article/view/14988/12377

Organização Mundial de Saúde (1997). Classificación internacionalde las deficiências, actividades e participación: um manual de las dimensiones de la inhabilitación e su funcionamento.

Sacks, L., Nakaji, M., Harry, K. M., Oen, M., Malcarne, V. L., \& Sadler, G. R. (2013). Testicular cancer knowledge among deaf and hearing men. Journal of cancer education, 28(3), 503-508. https://www-ncbi-nlm-nih.ez360.periodicos.capes.gov.br/pmc/articles/PMC3770485/

Soares, I. P., Lima, E. M. M., Santos, A. C. M., \& Ferreira, C. B. (2018). Como eu falo com você? a comunicação do enfermeiro com o usuário surdo. Revista Baiana de Enfermagem. 32, e25978. http://dx.doi.org/10.18471/rbe.v32.25978

Souza, M. T. D., Silva, M. D. D., \& Carvalho, R. D. (2010). Revisão integrativa: o que é e como fazer. Einstein, 8(1), $102-106$. https://www.scielo.br/scielo.php?pid=S1679-45082010000100102\&script=sci_arttext\&tlng=pt 